\title{
Utility of Immunofluorescence in the Diagnosis of Glomerulopathies
}

\section{Utilidad de la Inmunofluorescencia en el Diag- nóstico de las Glomerulopatías}

XVIII International Seminar on Health, Food and Human Nutrition

Corresponding Author:

Rolando Teruel Ginés

teruelcuba@gmail.com

Published: 9 September 2021

Production and Hosting by

Knowledge E

(c) Rolando Teruel Ginés et al. This article is distributed under the terms of the Creative Commons Attribution License, which permits unrestricted use and redistribution provided that the original author and source are credited.
Rolando Teruel Ginés ${ }^{1}$, lliana Ojeda Armas², María de los Ángeles Leyva Montero ${ }^{3}$, Fidel Enrique Infante Romero ${ }^{4}$, and Liliana Teruel Leyva ${ }^{4}$

${ }^{1}$ Departamento de Medicina Interna II, Facultad de Salud Pública, Escuela Superior Politécnica de Chimborazo, Chimborazo, Ecuador

${ }^{2}$ Departamento de Histología Humana II, Facultad de Salud Pública, Escuela Superior Politécnica de Chimborazo, Chimborazo, Ecuador

${ }^{3}$ Departamento de Histología Humana I y Patología Humana, Facultad de Salud Pública, Escuela Superior Politécnica de Chimborazo, Chimborazo, Ecuador

${ }^{4}$ Estudiantes de la Carrera de Medicina, Facultad de Salud Pública. Escuela Superior Politécnica de Chimborazo, Chimborazo, Ecuador

\section{Abstract}

Introduction: Glomerulopathies constitute aheterogeneous group of diseases that are difficult to diagnose and immunofluorescence is currently considered an invaluable technique to establish an adequate diagnosis and a correct therapeutic approach. Objective: To contribute to the updating of the scientific community in relation to the diagnosis of this pathology with the use of immunofluorescence. Methodology: A non-systematic bibliographic review was carried out in the databases available on the Internet such as: Scielo, Elsevier, Cochrane Library, PubMed, Intramed. This search was conducted in both Spanish and English. Results: 55 bibliographic sources were found, of which 30 relevant scientific articles were used and 25 were excluded because they did not meet the selection criteria. Discussion: Glomerulopathies comprise a group of diseases that affect renal function, they can be primary or secondary and have an acute or chronic and progressive course, with high morbidity and mortality worldwide, since they can lead patients to renal failure, Therefore, the use of diagnostic means such as immunofluorescence is an invaluable tool to achieve a specific diagnosis, which will result in an appropriate therapeutic approach for each patient. Conclusion: Immunofluorescence is an invaluable technique in the diagnosis of glomerulopathies that facilitates an adequate therapeutic approach for patients. And it allows to establish the specific type between the different variants of this group of diseases.

Keywords: glomerulopathies, immunofluorescence, renal failure, glomerular filtration, immune complex deposits.

\section{Resumen}

Introducción: Las glomerulopatías constituyen un grupo heterogéneo de enfermedades de difícil diagnóstico y la inmunofluorescencia es considerada en la actualidad una técnica de inestimable valor para establecer un diagnóstico adecuado y un correcto enfoque terapéutico. Objetivo: Contribuir a la actualización de la comunidad científica en relación al diagnóstico de esta patología con el uso de la inmunofluorescencia. Métodos: Se realizó una revisión bibliográfica no sistemática en las bases de datos disponibles de internet como: Scielo, Elsevier, Biblioteca Cochrane, PubMed, Intramed. Esta búsqueda se realizó tanto en español como en 
inglés. Resultados: Se encontraron 55 fuentes bibliográficas, de las cuales se utilizaron $\mathbf{3 0}$ artículos científicos de relevancia y se excluyeron 25 debido a que no llegaron a cumplir con los criterios de selección. Discusión: Las glomerulopatías comprenden un grupo de enfermedades que afectan la función renal, pueden ser primarias o secundarias y tener un curso agudo o crónico y progresivo, con una alta morbimortalidad a nivel mundial, ya que pueden llevar a los pacientes a la insuficiencia renal, por lo que el uso de los medios diagnósticos como la inmunofluorescencia es de gran utilidad para definir el tipo específico dentro de este grupo heterogéneo de dolencias, lo que redundará en una mayor calidad de la asistencia médica para cada paciente. Conclusiones: La inmunofluorescencia es una técnica de valor inestimable en el diagnóstico de las glomerulopatías que facilita un enfoque terapéutico adecuado para los pacientes. Y permite establecer el tipo específico entre las diferentes variantes de este grupo de enfermedades.

Palabras Clave: glomerulopatías, inmunofluorescencia, insuficiencia renal, filtrado glomerular, depósitos de inmunocomplejos.

\section{Introducción}

Las glomerulopatías comprenden un grupo de enfermedades que se caracterizan por una reducción del filtrado glomerular que puede progresar hacia la insuficiencia renal crónica; estas dolencias suponen algunos de los problemas más importantes y de difícil diagnóstico para la Nefrología ya que no siempre el paciente aqueja los síntomas del daño renal, puede sospecharse la enfermedad cuando un individuo presenta un uroanálisis de rutina con resultado anormal [1-4].

Los glomérulos pueden resultar afectados en diversas situaciones patológicas, en las cuales pueden estar imbricados numerosos factores, entre ellos se destacan los trastornos inmunitarios, los cuales son la base de la mayoría de las glomerulopatías primarias, y están presentes en muchas secundarias; estas patologías pueden tener un curso agudo o crónico, y en el caso de estas últimas el diagnóstico puede ser tardío, en una etapa donde ya el paciente puede ser portador de una insuficiencia renal [1, 3, 5-8].

El diagnóstico de este heterogéneo grupo de dolencias ha tenido un significativo avance debido al vertiginoso desarrollo de los medios diagnósticos en la medicina moderna, ya que, en la actualidad, con el uso de los estudios de imágenes y los métodos de la Anatomía Patológica, cada vez se diagnostica un mayor número de enfermos en una etapa donde existen mejores opciones de tratamiento que le aseguren una calidad de vida adecuada a estos pacientes [9-12].

Entre los estudios que se realizan para obtener un diagnóstico definitivo cada vez tiene mayor protagonismo el uso de la biopsia renal, la misma consiste en la toma de una muestra a través de una punción con aguja gruesa que atraviesa la piel a nivel de la región lumbar y penetra hasta el riñón para obtener un pequeño cilindro de tejido del parénquima renal, el cual luego de un procesamiento técnico es examinado por el patólogo con microscopía óptica, electrónica y de inmunofluorescencia [1, 13-15]. 
El examen minucioso de dicha muestra con cada uno de estos microscopios aportará elementos morfológicos importantes para establecer el diagnóstico de la glomerulopatía en cuestión, pero es la inmunofluorescencia, procedimiento que permite demostrar la fijación del anticuerpo al antígeno específico con marcado previo del anticuerpo con una sustancia fluorescente, lo que permite observar de manera específica los depósitos de inmunocomplejos con los diferentes patrones, los cuales son específicos para las diferentes variantes de glomerulopatías [1, 16, 17].

Teniendo en cuenta estos aspectos en el diagnóstico de estas enfermedades nos hemos motivado a realizar este trabajo de revisión para contribuir a la actualización de la comunidad científica en relación al diagnóstico de esta patología con el uso de la inmunofluorescencia.

\section{Metodología}

\subsection{Método de búsqueda bibliográfica}

Se realizó una investigación no sistemática de carácter descriptivo, tomando en cuenta las definiciones y la situación actual del tema a tratar.

\subsection{Bases de datos consultadas}

Las diferentes fuentes bibliográficas utilizadas para realizar este trabajo han sido buscadas y encontradas en internet, utilizando bases de datos como PubMed, Medline, publicaciones periódicas y Scielo; se verificó el año de publicación y datos de autor con el fin de seleccionar las más confiables. Además, para la selección de palabras clave utilizamos los Descriptores de en Ciencias de la Salud (DeCS), a través de la utilización de las palabras clave: Glomerulopatías, inmunofluorescencia, insuficiencia renal, filtrado glomerular, depósitos de inmunocomplejos.

\subsection{Criterios de inclusión y exclusión}

Para nuestra revisión, seleccionamos publicaciones de no más de 5 años de antigüedad, tomando prioritariamente artículos de información actualizada y novedosa, con el fin de obtener información real de la situación actual. Excluyendo aquellos artículos que tenían falta de datos del autor, al igual que en aquellos que no se tuvo acceso al artículo original completo.

\subsection{Idioma y fecha de búsqueda}

La bibliografía seleccionada fue buscada en idioma español e inglés, y publicada durante los últimos 5 años 


\section{Resultados}

Se revisaron 50 artículos científicos, de los cuales fueron seleccionados 30 de acuerdo con los autores y fecha de publicación, descartando 20, de los cuales 16 fue por falta de datos del autor y 4 porque no se pudo tener acceso al artículo original completo.

\section{Discusión}

La inmunofluorescencia no es más que una técnica de inmunomarcación, que al hacer uso de anticuerpos marcados químicamente con una sustancia fluorescente y que al mismo tiempo dicho anticuerpo tiene una afinidad específica para reaccionar con una determinada molécula presente en las secciones de tejido que están siendo estudiadas, que en este caso sería el antígeno, al formarse la unión antígeno-anticuerpo puede ser observado este fenómeno por la presencia de la sustancia fluorescente con el uso de un microscopio de inmunofluorescencia, lo que nos permite la detección de los inmunocomplejos en las glomerulopatías. Estos inmunocomplejos están formados por proteínas específicas como las inmunoglobulinas (lg), fragmentos del sistema del complemento y fibrina, las cuales están presentes en los mismos, teniendo un mayor significado para el diagnóstico en el caso de las enfermedades glomerulares primarias debido a que en estos casos, por lo general, no existe en el paciente una historia de enfermedad previa como ocurre en el caso de las secundarias, en las que los enfermos ya tienen antecedentes patológicos personales de alguna afección sistémica como por ejemplo: Lupus Eritematoso Sistémico (LES), Diabetes Mellitus (DM), Hipertensión Arterial (HTA), etc. [18-20].

Esta técnica ha sido usada hace más de 60 años por los investigadores, pero con el desarrollo de la Nefrología y los avances en el descubrimiento de la base inmunológica de la mayoría de estas afecciones, ha tomado auge en la práctica médica la utilización de la misma para conformar un diagnóstico más específico en cada tipo de glomerulopatía que puede presentar un paciente determinado [14, 18-20].

La interpretación por parte del patólogo del patrón de disposición de estos inmunocomplejos, su localización en las diferentes estructuras histológicas del corpúsculo renal y el tipo de anticuerpo que se utilice para el inmunomarcaje tendrá como resultado el diagnóstico definitivo. Los criterios inmunopatológicos que permiten establecer el tipo de enfermedad glomerular específica se basan en la demostración del tipo de inmunoglobulina que presentan estos depósitos y la forma en que se disponen, la presencia de fragmentos del complemento y en algunos casos otras proteínas como por ejemplo el amiloide, lo que evidencia la base inmunitaria del daño que se produce a nivel del glomérulo renal [5, 21-25].

Para lograr la observación de estos hallazgos morfológicos es necesario la utilización del microscopio de inmunofluorescencia, cuyo principio de funcionamiento se basa en la utilización de una fuente de luz de mercurio a alta presión que ilumina los cortes histológicos. Adicionalmente este equipo posee filtros especiales que permiten seleccionar la longitud de onda de los rayos luminosos que llegan a la muestra y también los que emite ésta, de manera tal que las sustancias fluorescentes que se usan para marcar 
los anticuerpos que se emplean en una muestra determinada se observan brillantes y con color, lo cual permite la localización en los tejidos de los inmunocomplejos, de esta forma podemos diagnosticar la posible causa de esta variedad de patologías [3, 21, 26-29].

En el diagnóstico de las lesiones glomerulares relacionadas con el síndrome de nefritis aguda, los hallazgos que evidencia el estudio de la biopsia renal con el uso de la inmunofluorescencia contribuyen a establecer la etiología del síndrome, por ejemplo cuando observamos depósitos inmunitarios granulares de $\operatorname{lgG}$, IgM y el fragmento C3 del complemento en el mesangio y a lo largo de las membranas basales glomerulares que pueden ser focales y escasos debe plantearse una glomerulonefritis aguda proliferativa y la etiología de esta debe ser post estreptocócica, mientras que si los depósitos son lineales puede tratarse del síndrome de Goodpasture; en los casos idiopáticos los depósitos pueden ser apenas visibles [21, 26].

Otro ejemplo de la utilidad de esta técnica para hacer el diagnóstico certero en estas patologías es cuando se estudia un síndrome nefrótico, el cual puede deberse a múltiples causas, y en cada una de ellas la inmunofluorescencia mostrará un patrón diferente de los depósitos inmunitarios. Dentro de las causas más frecuente de dicho síndrome en la infancia está le enfermedad por mínimos cambios, patología de curso relativamente benigno, en la cual aproximadamente el 10\% de los pacientes puede presentar recidivas y en estos casos puede estar indicada la biopsia renal, este espécimen es ampliamente estudiado y en este caso la inmunofluorescencia revela la ausencia de inmunocomplejos y de fragmentos del complemento, lo cual descarta la posibilidad de errores diagnósticos; en estos casos los pacientes suelen responder a la terapia con el uso de los esteroides a largo plazo y curan al llegar a la pubertad [12, 15, 18, 30].

La glomerulonefritis membranoproliferativa, representa un grupo de enfermedades que se caracterizan desde el punto de vista histopatológico por alteraciones en la membrana basal y proliferación de células glomerulares, la cual es predominantemente mesangial; este grupo de patologías representa del 5 al 10\% de todos los casos de síndrome nefrótico en niños y adultos, la mayoría de los casos son debidos a afectaciones idiopáticas del glomérulo, por lo que en los mismos se trata de una enfermedad primaria, aunque también se presentan casos por causas secundarias asociados a enfermedades sistémicas. Las primarias se clasifican, teniendo en cuenta las alteraciones ultraestructurales y de inmunofluorescencia, en dos tipos principales: Tipo I y tipo II, y en estos casos los hallazgos de inmunofluorescencia revelan la presencia inmunocomplejos en forma de depósitos granulares constituidos por lgG y fragmentos del complemento como C3, C1q y C4 en las lesiones de tipo I, mientras que en las lesiones de tipo Il sólo se observa la presencia del fragmento C3 del complemento que se dispone de forma granular o lineal [8, 25, 26, 30].

También la inmunofluorescencia puede aportar datos de interés en el diagnóstico de glomerulopatías secundarias, las cuales se presentan en el curso de enfermedades sistémicas, debidas a alteraciones inmunitarias, metabólicas o hereditarias, en las cuales puede demostrarse en el estudio histopatológico la presencia de inmunocomplejos constituidos por IgG e IgM como es el caso de LES, o de IgA, como sucede en la púrpura de Henoch Schönlein [1, 19, 20]. 


\section{Conclusiones}

La inmunofluorescencia es una técnica de valor inestimable en el diagnóstico de las glomerulopatías que facilita un enfoque terapéutico adecuado para los pacientes y permite establecer el tipo específico entre las diferentes variantes de este grupo de enfermedades.

\section{Agradecimientos}

Los autores agradecen a la Facultad de Salud Pública por dar la oportunidad de participar en este importante evento científico que constituirá un nuevo logro para nuestra institución.

\section{Conflicto de Intereses}

Los autores declaran no tener ningún conflicto de interés.

\section{Limitación de Responsabilidad}

Los puntos de vista tratados en la presente revisión bibliográfica se encuentran netamente bajo la responsabilidad

de los autores.

\section{Fuentes de Apoyo}

El desarrollo del presente trabajo fue producto de autofinanciación.

\section{References}

[1] Annamalai I, Chandramohan G, Srinivasa Prasad ND, Fernando E, Sujith S. Rapidly progressive glomerulonephritis due to anti-glomerular basement membrane disease accompanied by $\lg A$ nephropathy: An unusual association. Saudi J Kidney Dis Transplant Off Publ Saudi Cent Organ Transplant Saudi Arab. diciembre de 2017;28(6):1404-7.

[2] Deshpande NS, Tewari R, Badwal S, Mendonca S, Bharadwaj R. Evaluation of cases of membranoproliferative glomerulonephritis according to newer classification: A retrospective recordbased study. Med J Armed Forces India. julio de 2018;74(3):264-7.

[3] Guo H, Wang B, Li H, Ling L, Niu J, Gu Y. Glucagon-like peptide-1 analog prevents obesity-related glomerulopathy by inhibiting excessive autophagy in podocytes. Am J Physiol Renal Physiol. 01 de 2018;314(2):F181-9.

[4] Park JM, Lee H, Song S, et al. Primary Glomerulonephritis with Unique C4d Deposition and Concurrent Non-infectious Intermediate Uveitis: a Case Report and Literature Review. J Korean Med Sci. 30 de abril de 2018;33(18):e136.

[5] de Kort H, Willicombe M, Brookes P, et al. Peritubular Capillary Basement Membrane Multilayering in Renal Allograft Biopsies of Patients With De Novo Donor-Specific Antibodies. Transplantation. abril de 2016;100(4):889-97.

[6] Sethi S, Vrana JA, Fervenza FC, et al. Characterization of C3 in C3 glomerulopathy. Nephrol Dial Transplant Off Publ Eur Dial Transpl Assoc- Eur Ren Assoc. 01 de 2017;32(3):459-65. 
[7] Malvar A, Davila P, Ferrari M, et al. Podocyte infolding glomerulopathy; report of the first case in Latin America and review of the literature. Nefrol Publicacion Of Soc Espanola Nefrol. 14 de enero de 2020;

[8] Pirozzi N, Stoppacciaro A, Menè P. Dominant C3 glomerulopathy: new roles for an old actor in renal pathology. J Nephrol. 2018;31(4):503-10.

[9] García-Curdi F, Lois-Ortega Y, Gonzales-Sejas AG, Alfonso-Collado Jl, Fumanal-Senz L, Vallés-Varela $\mathrm{H}$. Enfermedad relacionada con IgG4 con afectación exclusiva nasosinusal: presentación de un caso clínico y revisión de la literatura. Rev Esp Patol. abril de 2019;52(2):125-9.

[10] Arapović A, Vukojević K, Filipović N, et al. Epidemiology of 10-year paediatric renal biopsies in the region of southern Croatia. BMC Nephrol. diciembre de 2020;21(1):1-12.

[11] Hashmi AA, Hussain Z, Edhi MM, Mumtaz S, Faridi N, Khan M. Insight to changing morphologic patterns of glomerulopathy in adult Pakistani patients: an institutional perspective. BMC Res Notes. 8 de febrero de 2016;9:73.

[12] Fukunaga M, Nagahama K, Aoki M, et al. Membranous Nephropathy- Like Apolipoprotein E Deposition Disease with Apolipoprotein E Toyonaka (Ser197Cys) and a Homozygous Apolipoprotein E2/2. Case Rep Nephrol Dial. abril de 2018;8(1):45-55.

[13] Pizzo HP, Haas M, Puliyanda D. Collagen type III glomerulopathy. Kidney Int. junio de 2018;93(6):1490.

[14] Dong J, Wei H, Han M, Guan Y, Wu Y, Li H. Collagen type III glomerulopathy: A case report and review of 20 cases. Exp Ther Med. octubre de 2015;10(4):1445-9.

[15] Stump M, Messingham KN, Fairley JA. Concurrent mucous membrane pemphigoid and membranous glomerulonephritis in a patient with autoantibodies targeting the 1080 region of collagen XVII. Br J Dermatol. 2019;181(4):835-6.

[16] Roy S, Nalwa A, Keith J, Weck K, Singh H, Nickeleit V. Pseudolinear C4d deposits in a hereditary glomerulopathy caused by a rare NC1 collagen-4-alpha-5 missense mutation: a «new disease entity»? Ultrastruct Pathol. 2019;43(4-5):209-15.

[17] Herrera GA. The value of ultrastructural evaluation in medical renal diseases. Ultrastruct Pathol. 2 de noviembre de 2019;43(6):225-8.

[18] Hirashima H, Komiya T, Toriu N, et al. A case of nephrotic syndrome showing contemporary presence of apolipoprotein E2 homozygote glomerulopathy and membranous nephropathy-like findings modified by apolipoprotein E Toyonaka. Clin Nephrol Case Stud. 2018;6:45-51.

[19] Kudose S, Santoriello D, Bomback AS, Stokes MB, D’Agati VD, Markowitz GS. Sensitivity and Specificity of Pathologic Findings to Diagnose Lupus Nephritis. Clin J Am Soc Nephrol CJASN. 7 de noviembre de 2019;14(11):1605-15.

[20] Larsen CP, Sharma SG, Caza TN, et al. Serum amyloid P deposition is a sensitive and specific feature of membranous-like glomerulopathy with masked IgG kappa deposits. Kidney Int. marzo de 2020;97(3):602-8.

[21] Wong A, Cianciolo RE. Comparison of immunohistochemistry and immunofluorescence techniques using anti- lambda light chain antibodies for identification of immune complex deposits in canine renal biopsies. J Vet Diagn Investig Off Publ Am Assoc Vet Lab Diagn Inc. septiembre de 2018;30(5):721-7.

[22] Bouatou Y, Kers J, Chevalier-Florquin MSN, et al. Diagnostic accuracy of immunofluorescence versus immunoperoxidase staining to distinguish immune complex-mediated glomerulonephritis and $\mathrm{C} 3$ dominant glomerulopathy. Histopathology. marzo de 2018;72(4):601-8.

[23] Nasr SH, Fidler ME, Said SM. Paraffin Immunofluorescence: A Valuable Ancillary Technique in Renal Pathology. Kidney Int Rep. noviembre de 2018;3(6):1260-6.

[24] Vessieres F, Cianciolo RE, Gkoka ZG, et al. Occurrence, management and outcome of immune-complex glomerulonephritis in dogs with suspected glomerulopathy in the UK. J Small Anim Pract. noviembre de 2019;60(11):683-90.

[25] Gong J, Zhan H, Li Y, Zhang W, Jin J, He Q. Krüppel-like factor 4 ameliorates diabetic kidney disease by activating autophagy via the mTOR pathway. Mol Med Rep. octubre de 2019;20(4):3240-8.

[26] Abd El hafez A, Hassan El-Esawy B, Mamdouh Abdelaziz A, Alaa Saleh M. Factors affecting the distribution of glomerulonephropathies among adult Saudi patients: A single-center, biopsy-based clinico-pathological study. IP Arch Cytol Histopathol Res. 28 de octubre de 2019;4(3):179-86.

[27] Rosenberg AZ, Kopp JB. Focal Segmental Glomerulosclerosis. Clin J Am Soc Nephrol CJASN. 7 de marzo de 2017;12(3):502-17.

[28] Filippone EJ, McCue PA, Farber JL. Transplant glomerulopathy. Mod Pathol Off J U S Can Acad Pathol Inc. 2018;31(2):235-52.

[29] Varshavsky VA, Grishina AN, Chebotareva NV, Gudkova El. [The clinical and morphological characteristics of C1q glomerulopathy]. Arkh Patol. 2018;80(1):46-51.

[30] Dinh A, Cuker A, Song W-C, Hogan JJ. Monoclonal immunoglobulin-associated C3 glomerulopathy and thrombotic microangiopathy: A real association? J Onco-Nephrol. 1 de febrero de 2019;3(1):3-10. 